\title{
Dynamic DEA: A slacks-based measure approach*
}

\author{
Kaoru Tone ${ }^{\mathrm{a}}$, Miki Tsutsui ${ }^{\mathrm{b}}$ \\ ${ }^{a}$ National Graduate Institute for Policy Studies, 7-22-1 Roppongi, Minato-ku, Tokyo 106-8677, \\ Japan \\ ${ }^{\mathrm{b}}$ Central Research Institute of Electric Power Industry, 2-11-2 Iwado Kita, Komae-shi, Tokyo \\ 201-8511, Japan
}

\begin{abstract}
In data envelopment analysis, there are several methods for measuring efficiency change over time, e.g. the window analysis and the Malmquist index. However, they usually neglect carry-over activities between consecutive two terms. These carry-overs play an important role in measuring the efficiency of decision making units in each term as well as over the whole terms based on the long-term viewpoint. Dynamic DEA model proposed by Färe and Grosskopf is the first innovative contribution for such purpose. In this paper we develop their model in the slacks-based measure (SBM) framework, called Dynamic SBM (DSBM). The SBM model is non-radial and can deal with inputs/outputs individually, contrary to the radial approaches that assume proportional changes in inputs/outputs. Furthermore, according to the characteristics of carry-overs, we classify them into four categories, i.e. desirable, undesirable, free and fixed. Desirable carry-overs correspond, for example, to profit carried forward and net earned surplus carried to the next term, while undesirable carry-overs include, for example, loss carried forward, bad debt and dead stock. Free and fixed carry-overs indicate, respectively, discretionary and non-discretionary ones. We develop Dynamic SBM models that can evaluate the overall efficiency of decision making units for the whole terms as well as the term efficiencies.
\end{abstract}

Keywords DEA, Dynamic DEA, DSBM, Carry-over

\section{Introduction}

Measurement of intertemporal efficiency change has long been a subject of concern in DEA. The window analysis by Klopp [11] was the first approach for this purpose (see also [2]). Based on Malmquist [12], Färe et al. [5] developed the Malmquist index in the DEA framework. This model can decompose the intertemporal efficiency change into catch-up and innovation (frontier-shift) effects.

However, these models do not account for the effect of carry-over activities between two consecutive terms. For each term these models have inputs and outputs but the connecting activities

\footnotetext{
* Research supported by Grant-in-Aid for Scientific Research, Japan Society for the Promotion of Science.
} 
between terms are not accounted for explicitly.

The dynamic DEA model proposed by Färe and Grosskopf [4] is the first innovative scheme for dealing formally with these inter-connecting activities. See also [13, 14, 16, 1 and 10] for further references.

In this paper, we extend their model within the slacks-based measure framework proposed by Tone [17] and Pastor et al. [15]. Hence, our model is non-radial and can deal with inputs/outputs individually, which enable us to obtain non-uniform input/output factor efficiencies, contrary to the radial approaches that assume proportional changes in inputs/outputs and provide only uniform input/output factor efficiency. We can put weights to input/output items according to their importance. Furthermore, we categorize the carry-overs, called links, into four types; desirable (good), undesirable (bad), discretionary (free) and non-discretionary (fixed), reflecting actual characteristics of carry-over activities. As for orientations of the model, we have three types; input-, output- and non-oriented. This paper can be positioned as an extension of the network SBM in Tone and Tsutsui [18] to the dynamic structure.

The rest of this paper unfolds as follows. In Section 2, we describe our dynamic model (DSBM). Links and their characteristics are discussed in Section 3, while production possibility set and models are presented in Section 4. Our objective functions and efficiency along with projections are presented in Section 5. In Section 6 we exhibit an empirical study on the generation division of fifty U.S.-Japan electric utilities. We also compare our results with the traditional separation model which deals with time series data year by year. We conclude this paper in the last section.

\section{Dynamic structure}

We observe the dynamic structure exhibited in Figure 1. We observe $n$ DMUs (decision making units) over $T$ terms. At each term $t$, each DMU has its own inputs and outputs along with the carry-over (link) to the next term $t+1$. At the initial term 1 , we have no carry-over from the previous term and, at the terminal term $T$, we assume no carry-over to the next term.

\section{<<Figure 1: Dynamic structure >>}

\section{Links (carry-over) and their characteristics}

We classify carry-over activities, called links, into four categories as follows:

(1) Desirable (good) link

This indicates desirable carry-over, e.g. profit carried forward and net earned surplus carried to the next term. In our model, desirable links are treated as outputs and link value is restricted to be not less than the observed one. Comparative shortage of links in this category is accounted as inefficiency. 
(2) Undesirable (bad) link

This belongs to undesirable carry-over, e.g. loss carried forward, bad debt and dead stock. In our model, undesirable links are treated as inputs and its value is restricted to be not greater than the observed one. Comparative excess in links in this category is accounted as inefficiency.

(3) Discretionary (free) link

This corresponds to carry-over that DMU can handle freely. Its value can be increased or decreased from the observed one. The deviation from the current value is not directly reflected in the efficiency evaluation, but the continuity condition between two terms explained in the next section exerts an indirect effect on the efficiency score.

(4) Non-discretionary (fixed) link

This indicates carry-over that is beyond the control of DMU. Its value is fixed at the observed level. Similarly to free link, fixed link affects the efficiency score indirectly through the continuity condition between two terms.

\section{Production possibility set and models}

We deal with $n$ DMUs $(j=1, \ldots, n)$ over $T$ terms $(t=1, \ldots, T)$. At each term, DMUs have common $m$ inputs $(i=1, \mathrm{~K}, m), p$ non-discretionary (fixed) inputs $(i=1, \mathrm{~K}, p), s$ outputs $(i=1, \mathrm{~K}, s)$ and $r$ non-discretionary (fixed) outputs $(i=1, \mathrm{~K}, r)$. Let $x_{i j t}(i=1, \mathrm{~K}, m), x_{i j t}^{f i x}(i=1, \mathrm{~K}, p)$, $y_{i j t}(i=1, \mathrm{~K}, s)$ and $y_{i j t}^{f i x}(i=1, \mathrm{~K}, r)$ denote the observed (discretionary) input, non-discretionary input, (discretionary) output and non-discretionary output values of DMU $j$ at term $t$, respectively. We symbolize the four category links as $z^{\text {good }}, z^{\text {bad }}, z^{\text {free }}$ and $z^{\text {fix }}$. In order to identify them by term $(t)$, DMU (j) and item (i), we employ the notation $z_{i j t}^{\text {good }}(i=1, \mathrm{~K}, n g o o d ; j=1, \mathrm{~K}, n ; t=1, \mathrm{~K}, T)$ and etc. for denoting link values where ngood is the number of good links. These are all observed values up to the term $T$.

The production possible $\left\{x_{i t}\right\},\left\{x_{i t}^{\text {fix }}\right\},\left\{y_{i t}\right\},\left\{y_{i t}^{\text {fix }}\right\},\left\{z_{\text {it }}^{\text {good }}\right\},\left\{z_{i t}^{\text {bad }}\right\},\left\{z_{i t}^{\text {free }}\right\}$ and $\left\{z_{i t}^{\text {fix }}\right\}$ are defined by 


$$
\begin{aligned}
& x_{i t} \geq \sum_{j=1}^{n} x_{i j t} \lambda_{j}^{t} \quad(i=1, \mathrm{~K}, m ; t=1, \mathrm{~K}, T) \\
& x_{i t}^{f i x}=\sum_{j=1}^{n} x_{i j t}^{f i x} \lambda_{j}^{t} \quad(i=1, \mathrm{~K}, p ; t=1, \mathrm{~K}, T) \\
& y_{i t} \leq \sum_{j=1}^{n} y_{i j t} \lambda_{j}^{t} \quad(i=1, \mathrm{~K}, s ; t=1, \mathrm{~K}, T) \\
& y_{i t}^{\text {fix }}=\sum_{j=1}^{n} y_{i j t}^{f i x} \lambda_{j}^{t} \quad(i=1, \mathrm{~K}, r ; t=1, \mathrm{~K}, T) \\
& z_{i t}^{\text {good }} \leq \sum_{j=1}^{n} z_{i j t}^{\text {good }} \lambda_{j}^{t} \quad(i=1, \mathrm{~K}, \text { ngood } ; t=1, \mathrm{~K}, T) \\
& z_{i t}^{\text {bad }} \geq \sum_{j=1}^{n} z_{i j t}^{\text {bad }} \lambda_{j}^{t} \quad(i=1, \mathrm{~K}, n b a d ; t=1, \mathrm{~K}, T) \\
& z_{i t}^{\text {free }}: \text { free } \quad(i=1, \mathrm{~K}, \text { nfree } ;=1, \mathrm{~K}, T) \\
& z_{i t}^{\text {fix }}=\sum_{j=1}^{n} z_{i j t}^{\text {fix }} \lambda_{j}^{t} \quad(i=1, \mathrm{~K}, n f i x ; t=1, \mathrm{~K}, T) \\
& \sum_{j=1}^{n} \lambda_{j}^{t}=1 \quad(t=1, \mathrm{~K}, T)
\end{aligned}
$$

where $\lambda^{t} \in R^{n}(t=1, \mathrm{~K}, T)$ is the intensity vector for the term $t$. The last constraint corresponds to the variable returns-to-scale assumption. If we delete this constraint, we have the constant returns-to-scale model.

The continuity of link flows between terms $t$ and $t+1$ can be guaranteed by the following condition:

$$
\sum_{j=1}^{n} z_{i j t}^{\alpha} \lambda_{j}^{t}=\sum_{j=1}^{n} z_{i j t}^{\alpha} \lambda_{j}^{t+1} \quad(\forall i ; t=1, \mathrm{~K}, T-1)
$$

where the symbol $\alpha$ stands for good, bad, free or fix.

Using these expressions for production, we can express $\operatorname{DMU}_{o}(o=1, \ldots, n)$ as follows:

$$
\begin{aligned}
& x_{i o t}=\sum_{j=1}^{n} x_{i j t} \lambda_{j}^{t}+s_{i t}^{-} \quad(i=1, \mathrm{~K}, m ; t=1, \mathrm{~K}, T) \\
& x_{i o t}^{f i x}=\sum_{j=1}^{n} x_{i j t}^{f i x} \lambda_{j}^{t} \quad(i=1, \mathrm{~K}, p ; t=1, \mathrm{~K}, T) \\
& y_{i o t}=\sum_{j=1}^{n} y_{i j t} \lambda_{j}^{t}-s_{i t}^{+} \quad(i=1, \mathrm{~K}, s ; t=1, \mathrm{~K}, T) \\
& y_{i o t}^{f i x}=\sum_{j=1}^{n} y_{i j t}^{\text {fix }} \lambda_{j}^{t} \quad(i=1, \mathrm{~K}, r ; t=1, \mathrm{~K}, T) \\
& z_{i o t}^{\text {good }}=\sum_{j=1}^{n} z_{i j t}^{\text {good }} \lambda_{j}^{t}-s_{i t}^{\text {good }} \quad(i=1, \mathrm{~K}, \text { ngood } ; t=1, \mathrm{~K}, T) \\
& z_{\text {iot }}^{\text {bad }}=\sum_{j=1}^{n} z_{i j t}^{\text {bad }} \lambda_{j}^{t}+s_{i t}^{\text {bad }} \quad(i=1, \mathrm{~K}, n b a d ; t=1, \mathrm{~K}, T) \\
& z_{\text {iot }}^{\text {free }}=\sum_{j=1}^{n} z_{i j t}^{\text {free }} \lambda_{j}^{t}+s_{i t}^{\text {free }} \quad(i=1, \mathrm{~K}, n f r e e ; t=1, \mathrm{~K}, T) \\
& z_{i o t}^{\text {fix }}=\sum_{j=1}^{n} z_{i j t}^{\text {fix }} \lambda_{j}^{t} \quad(i=1, \mathrm{~K}, n f i x ; t=1, \mathrm{~K}, T) \\
& \sum_{j=1}^{n} \lambda_{j}^{t}=1 \quad(t=1, \mathrm{~K}, T) \\
& s_{i t}^{-} \geq 0, s_{i t}^{+} \geq 0, s_{i t}^{\text {good }} \geq 0, s_{i t}^{\text {bad }} \geq 0 \text { and } s_{i t}^{\text {free }}: \text { free }(\forall i, t)
\end{aligned}
$$


where $s_{i t}^{-}, s_{i t}^{+}, s_{i t}^{\text {good }}, s_{i t}^{\text {bad }}$ and $s_{i t}^{\text {free }}$ are slacks denoting, respectively, input excess, output shortfall, link shortfall, link excess and link deviation.

\section{Objective functions and efficiency}

We evaluate the overall efficiency of $\mathrm{DMU}_{o} \quad(o=1, \ldots, n)$ taking $\left(\left\{\lambda^{t}\right\},\left\{\mathbf{s}_{t}^{-}\right\},\left\{\mathbf{s}_{t}^{+}\right\},\left\{\mathbf{s}_{t}^{\text {good }}\right\},\left\{\mathbf{s}_{t}^{\text {bad }}\right\},\left\{\mathbf{s}_{t}^{\text {free }}\right\},\left\{\mathbf{s}_{t}^{\text {fix }}\right\}\right)$ as variables, in the following three orientations.

\subsection{Input-oriented case}

The input-oriented overall efficiency $\theta_{o}^{*}$ is defined by

$$
\theta_{o}^{*}=\min \frac{1}{T} \sum_{t=1}^{T} w^{t}\left[1-\frac{1}{m+n b a d}\left(\sum_{i=1}^{m} \frac{w_{i}^{-} s_{i t}^{-}}{x_{i o t}}+\sum_{i=1}^{n b a d} \frac{s_{i t}^{\text {bad }}}{z_{i o t}^{\text {bad }}}\right)\right]
$$

subject to (2) and (3), where $w^{t}$ and $w_{i}^{-}$are weights to term $t$ and input $i$ which are supplied exogenously according to their importance and satisfy the conditions.

$$
\sum_{t=1}^{T} w^{t}=T \text { and } \sum_{i=1}^{m} w_{i}^{-}=m .
$$

This model deals with excesses in input resources and undesirable (bad) links as main targets of evaluation.

Let an optimal solution of (4) be $\left(\left\{\lambda_{o}^{t^{*}}\right\},\left\{\mathbf{s}_{o t}^{-*}\right\},\left\{\mathbf{s}_{o t}^{+*}\right\},\left\{\mathbf{s}_{o t}^{\text {good** }}\right\},\left\{\mathbf{s}_{o t}^{\text {bad }^{*}}\right\},\left\{\mathbf{s}_{o t}^{\text {free* }}\right\},\left\{\mathbf{s}_{o t}^{\text {fi** }}\right\}\right)$. We define the term efficiency $\theta_{o t}$ by

$$
\theta_{o t}=1-\frac{1}{m+n b a d}\left(\sum_{i=1}^{m} \frac{w_{i}^{-} s_{\text {iot }}^{-*}}{x_{\text {iot }}}+\sum_{i=1}^{\text {nbad }} \frac{s_{i o t}^{\text {bad* }}}{z_{\text {iot }}^{\text {bad }}}\right) \quad(t=1, \mathrm{~K}, T)
$$

This term efficiency expresses the input-oriented efficiency score for the term. The overall efficiency is the weighted average of the term efficiencies. Although the overall efficiency is uniquely determined as the optimum value of the above LP, the term efficiency may have multiple optima. In order to find its range of variation, we can solve $\max (\min ) \theta_{o t}$, while keeping $\theta_{o}^{*}$ at the optimum.

[Definition 1] (Term input-efficiency)

If $\theta_{o t}=1, \mathrm{DMU}_{o}$ is called term input-efficient for the term $t$.

This implies that the optimal slacks in (6) are all zero, i.e. $s_{i o t}^{-*}=0(\forall i)$ and $s_{i o t}^{b a d^{*}}=0(\forall i)$. [Definition 2] (Overall input-efficiency)

If $\theta_{o}^{*}=1, \mathrm{DMU}_{o}$ is called overall input-efficient.

Thus, this means $s_{i o t}^{-*}=0(\forall i, t)$ and $s_{i o t}^{b a d^{*}}=0(\forall i, t)$. 


\section{[Theorem 1]}

$\mathrm{DMU}_{o}$ is overall input-efficient, if and only if it is term input-efficient for all terms.

\subsection{Output-oriented case}

The output-oriented overall efficiency $\tau_{o}^{*}$ is defined by

$$
1 / \tau_{o}^{*}=\max \frac{1}{T} \sum_{t=1}^{T} w^{t}\left[1+\frac{1}{s+n g o o d}\left(\sum_{i=1}^{s} \frac{w_{i}^{+} s_{i t}^{+}}{y_{\text {iot }}}+\sum_{i=1}^{\text {ngood }} \frac{s_{i t}^{\text {good }}}{z_{\text {iot }}^{\text {good }}}\right)\right]
$$

subject to (2) and (3) where $w_{i}^{+}$is the weight to output $i$ and satisfies the condition.

$$
\sum_{i=1}^{s} w_{i}^{+}=s
$$

This model deals with shortfalls in output products and desirable (good) links as main targets of evaluation.

Using an optimal solution $\left(\left\{\lambda_{o}^{t^{*}}\right\},\left\{\mathbf{s}_{o t}^{-*}\right\},\left\{\mathbf{s}_{o t}^{+*}\right\},\left\{\mathbf{s}_{o t}^{\text {good** }}\right\},\left\{\mathbf{s}_{o t}^{\text {bad }^{*}}\right\},\left\{\mathbf{s}_{o t}^{\text {free* }}\right\},\left\{\mathbf{s}_{o t}^{\text {fix }}\right\}\right)$ to (7) we define the output-oriented term efficiency by

$$
\tau_{o t}=\frac{1}{1+\frac{1}{s+\text { ngood }}\left(\sum_{i=1}^{s} \frac{w_{i}^{+} s_{i o t}^{+*}}{y_{\text {iot }}}+\sum_{i=1}^{\text {ngood }} \frac{s_{\text {iot }}^{\text {good }}}{z_{\text {iot }}^{\text {good }}}\right)} \quad(t=1, \mathrm{~K}, T)
$$

The output-oriented overall efficiency $\tau_{o}^{*}$ is the weighted harmonic mean of the term efficiencies.

\section{[Definition 3] (Term output-efficiency)}

If $\tau_{o t}=1, \mathrm{DMU}_{o}$ is called term output-efficient for the term $t$.

This implies that the optimal slacks in (9) are all zero, i.e. $s_{i o t}^{+^{*}}=0(\forall i)$ and $s_{i o t}^{\text {good }}=0(\forall i)$.

[Definition 4] (Overall output-efficiency)

If $\tau_{o}^{*}=1, \mathrm{DMU}_{o}$ is called overall output-efficient.

This means $s_{i o t}^{+*}=0(\forall i, t)$ and $s_{i o t}^{\text {good }}{ }^{*}=0(\forall i, t)$.

\section{[Theorem 2]}

$\mathrm{DMU}_{o}$ is overall output-efficient, if and only if it is term output-efficient for all terms.

\subsection{Non-oriented case}

As the combination of input- and output-oriented cases, we define the non-oriented efficiency measure by solving program below: 


$$
\rho_{o}^{*}=\min \frac{\frac{1}{T} \sum_{t=1}^{T} w^{t}\left[1-\frac{1}{m+n b a d}\left(\sum_{i=1}^{m} \frac{w_{i}^{-} s_{i t}^{-}}{x_{i o t}}+\sum_{i=1}^{n b a d} \frac{s_{i t}^{\text {bad }}}{z_{i o t}^{\text {bad }}}\right)\right]}{\frac{1}{T} \sum_{t=1}^{T} w^{t}\left[1+\frac{1}{s+\text { ngood }}\left(\sum_{i=1}^{s} \frac{w_{i}^{+} s_{i t}^{+}}{y_{\text {iot }}}+\sum_{i=1}^{\text {goood }} \frac{s_{i t}^{\text {good }}}{z_{\text {iot }}^{\text {good }}}\right)\right]}
$$

subject to (2) and (3)..

This model deals with excesses in input resources and undesirable (bad) links and shortfalls in output products and desirable (good) links in a single unified scheme.

Using an optimal solution $\left(\left\{\lambda_{o}^{t^{*}}\right\},\left\{\mathbf{s}_{o t}^{\mathbf{s}^{*}}\right\},\left\{\mathbf{s}_{o t}^{+*}\right\},\left\{\mathbf{s}_{o t}^{\text {good*}}\right\},\left\{\mathbf{s}_{o t}^{\text {bad }}\right\},\left\{\mathbf{s}_{o t}^{\text {free* }}\right\},\left\{\mathbf{s}_{o t}^{f i x^{*}}\right\}\right)$ to (10) we define the non-oriented term efficiency by

$$
\rho_{o t}=\frac{1-\frac{1}{m+n b a d}\left(\sum_{i=1}^{m} \frac{w_{i}^{-} s_{i o t}^{-*}}{x_{i o t}}+\sum_{i=1}^{n b a d} \frac{s_{i o t}^{\text {bad }}}{z_{i o t}^{b a d}}\right)}{1+\frac{1}{s+\text { ngood }}\left(\sum_{i=1}^{s} \frac{w_{i}^{+} s_{i o t}^{+*}}{y_{i o t}}+\sum_{i=1}^{n g o o d} \frac{s_{i o t}^{\text {good }}}{z_{\text {iot }}^{\text {good }}}\right)}(t=1, \mathrm{~K}, T)
$$

[Definition 5] (Term efficiency)

If $\rho_{o t}=1, \mathrm{DMU}_{o}$ is called term efficient for the term $t$.

This implies that the optimal slacks in (11) are all zero, i.e. $s_{\text {iot }}^{-*}=0(\forall i), s_{i o t}^{\text {bad }}=0(\forall i), s_{i o t}^{+*}=0(\forall i)$ and $s_{\text {iot }}^{\text {good }}=0(\forall i)$.

[Definition 6] (Overall output-efficiency)

If $\rho_{o}^{*}=1, \mathrm{DMU}_{o}$ is called overall efficient.

This means $s_{i o t}^{-*}=0(\forall i, t), s_{i o t}^{b a d *}=0(\forall i, t), s_{i o t}^{+*}=0(\forall i, t)$ and $s_{i o t}^{\text {good }}{ }^{*}=0(\forall i, t)$.

[Theorem 3]

$\mathrm{DMU}_{o}$ is overall efficient, if and only if it is term efficient for all terms.

\subsection{Projection}

Let an optimal solution of (4), (7) or (10) be $\left(\left\{\lambda_{o}^{* *}\right\},\left\{\mathbf{s}_{o t}^{\mathbf{s}^{*}}\right\},\left\{\mathbf{s}_{o t}^{+*}\right\},\left\{\mathbf{s}_{o t}^{\text {good* }}\right\},\left\{\mathbf{s}_{o t}^{\text {bad }}\right\},\left\{\mathbf{s}_{o t}^{\text {free* }}\right\},\left\{\mathbf{s}_{o t}^{f i x^{*}}\right\}\right)$. We define the projection of $\mathrm{DMU}_{o}$ as follows: 


$$
\begin{aligned}
& \bar{x}_{i o t}=x_{i o t}-s_{i o t}^{-*} \quad(i=1, \mathrm{~K}, m ; t=1, \mathrm{~K}, T) \\
& \bar{x}_{\text {iot }}^{\text {fix }}=x_{\text {iot }}^{\text {fix }} \quad(i=1, \mathrm{~K}, p ; t=1, \mathrm{~K}, T) \\
& \bar{y}_{\text {iot }}=y_{\text {iot }}+s_{\text {iot }}^{+*} \quad(i=1, \mathrm{~K}, s ; t=1, \mathrm{~K}, T) \\
& \bar{y}_{\text {iot }}^{f i x}=y_{\text {iot }}^{f i x} \quad(i=1, \mathrm{~K}, r ; t=1, \mathrm{~K}, T) \\
& \bar{Z}_{\text {iot }}^{\text {good }}=z_{\text {iot }}^{\text {good }}+s_{\text {iot }}^{\text {good }} \quad(i=1, \mathrm{~K}, \text { ngood } ; t=1, \mathrm{~K}, T) \\
& \bar{Z}_{\text {iot }}^{\text {bad }}=z_{\text {iot }}^{\text {bad }}-s_{\text {iot }}^{\text {bad* }} \quad(i=1, \mathrm{~K}, \text { nbad } ; t=1, \mathrm{~K}, T) \\
& \bar{z}_{\text {iot }}^{\text {free }}=z_{\text {iot }}^{\text {free }}-s_{\text {iot }}^{\text {free* }} \quad(i=1, \mathrm{~K}, \text { nfree; } t=1, \mathrm{~K}, T) \\
& \bar{Z}_{\text {iot }}^{-f i x}=z_{\text {iot }}^{f i x} \quad(i=1, \mathrm{~K}, n f i x ; t=1, \mathrm{~K}, T)
\end{aligned}
$$

\section{[Theorem 4]}

The projected $\mathrm{DMU}_{o}$ is overall-efficient.

Proof.: We prove the theorem in the input-oriented case.

We evaluate the overall-efficiency of the projected DMU. Let an optimal solution be

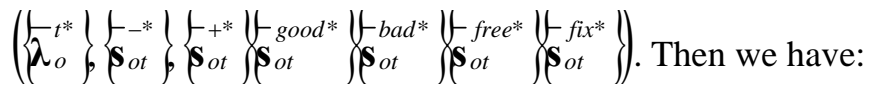

$$
\bar{x}_{i o t}=\sum_{j=1}^{n} x_{i j t} \bar{\lambda}_{j}^{t^{*}}+\bar{S}_{i o t}^{-*}(i=1, \mathrm{~K} \mathrm{~m}), \bar{z}_{i t o}^{\text {bad }}=\sum_{j=1}^{n} z_{i j t}^{\text {bad }} \bar{\lambda}_{j}^{t^{*}}+\bar{S}_{i o t}^{\text {bad* }}(i=1, \mathrm{~K}, \text { nbad }) .
$$

Replacing $\bar{X}_{\text {iot }}(i=1, \mathrm{~K} \mathrm{~m}), \bar{Z}_{\text {ito }}^{\text {bad }}(i=1, \mathrm{~K}$, nbad $)$ by (12), we have:

$$
x_{i o t}=\sum_{j=1}^{n} x_{i j t} \bar{\lambda}_{j}^{t^{*}}+\bar{S}_{i o t}^{-*}+s_{i o t}^{-*}(i=1, \mathrm{~K} \mathrm{~m}), z_{i t o}^{b a d}=\sum_{j=1}^{n} z_{i j t}^{b a d} \bar{\lambda}_{j}^{t^{*}}+\bar{S}_{i o t}^{b a d^{*}}+s_{i o t}^{b a d^{*}}(i=1, \mathrm{~K}, \text { nbad }) .
$$

Corresponding to this expression we have the overall-efficiency

$$
\bar{\theta}_{o}^{*}=\frac{1}{T} \sum_{t=1}^{T} w^{t}\left[1-\frac{1}{m+n b a d}\left(\sum_{i=1}^{m} \frac{w_{i}^{-}\left(s_{i o t}^{-*}+\bar{S}_{i o t}^{-*}\right)}{x_{i o t}}+\sum_{i=1}^{n b a d} \frac{S_{i o t}^{b a d^{*}}+\bar{S}_{\text {iot }}}{z_{\text {iot }}^{\text {bad }}}\right)\right] .
$$

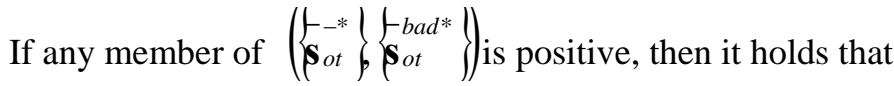

$$
\bar{\theta}_{o}^{*}<\theta_{o}^{*} \text {. }
$$

This contradicts the optimality of $\theta_{o}^{*}$. Thus, we have $\stackrel{-\mathbf{S}_{o t}^{*}}{=\mathbf{0}}$ and $\stackrel{-\mathbf{S}_{o t}}{\mathbf{b}^{*}}=\mathbf{0}$. Hence, the projected DMU is overall-efficient.

Similarly, we can prove the theorem in the output-oriented and non-oriented models. Q.E.D. 


\section{An empirical study: Application to electric utilities in U.S. and Japan}

We applied our dynamic SBM model to evaluate efficiency change over time regarding the power generation division of fifty electric utilities consisting of 41 U.S. and 9 Japanese companies.

\subsection{Data}

We utilized the data set comprised of 41 U.S. and 9 Japan electric utilities for 7 years: 1990-1996. For each DMU and for each year we used two inputs; the number of employees and consumed fuel in generation division, and a single output; generated power. As the link, we employed the generation capacity as capital asset at the end of each year, which will carry-over to the next year.

Electric power industry is a typical capital intensive industry, and thus it is essential for utilities to have a long-term view for asset management. Actually, they invest in their facilities to cover electricity demands over the next several years. From this view point, methods to measure management efficiency for each year can be biased. To cope with the long-term investment policy in this industry, dynamic DEA models must be suitable.

The Japanese dataset for this study was obtained from the "Handbook of Electric Power Industry" published by the Federation of Electric Power Companies (FEPC) in Japan [6], while the U.S. dataset was constructed from the "FORM No.1" and "FORM No.423" published by the Federal Energy Regulatory Commission (FERC) [7 and 8] and "Form EIA-860" published by Energy Information Administration (EIA) [3]. Table 1 exhibits statistics of the data set.

\section{$<<$ Table 1: Statistics of data set $>>$}

\subsection{Model}

We used the non-oriented and constant returns-to-scale (CRS) model. We assumed four characteristics of link (capital asset) in order to compare the results. In the desirable link case, capital asset is treated as output, whereas in the undesirable case it is treated as input. As the weights $w^{t}$, $w_{i}^{+}$and $w_{i}^{-}$in Equations (4, 7 and 10), we employed $w^{t}=1(\forall t)$ and $w_{i}^{+}=w_{i}^{-}=1 \quad(\forall i)$.

\subsection{Results}

Figure 2 reports the averages of efficiency of 50 DMUs for years 1990-1996.

\section{<<Figure 2: Averages of efficiency change of 50 US and Japanese DMUs >>}

Under any scenario on links, i.e. desirable (as output), undesirable (as input), free and fix, the time trend of average efficiencies is increasing. However, focusing on respective DMUs, it is observed that efficiency trends vary depending on the definition of the link variable. Figures 3 and 5 
depict the results of DMUs A and B, respectively, and Figures 4 and 6 indicate the inverse of the capital productivity index (ICPI) for these two DMUs and the average for 50 DMUs. ICPI is calculated as the amount of capital input divided by the amount of output. ${ }^{1}$ DMU with high ICPI score is less productive in spending large amount of capital inputs relative to the generated power.

$<<$ Figure 3: Efficiency change of DMU A >>

$<<$ Figure 4: Comparison of the inverse of capital productivity index between DMU A and the average of 50 DMUs >>

$<<$ Figure 5: Efficiency change of DMU B $>>$

$<<$ Figure 6: Comparison of the inverse of capital productivity index between DMU B and the average of 50 DMUs $>>$

In this study, the capital asset is utilized as a carried-over link variable. The DMU A carried over the large capital asset relative to its generated power (see Figure 4), which is highly valued in the desirable (as output) model as shown in Figure 3. Contrary to this, it is regarded as the excess input resulting in inefficiency in the undesirable (as input) model. The DMU B is in the opposite case, i.e. small carry-over relative to its output (Figure 6) makes this DMU to be efficient in the 'as input' model, while it lowers the efficiency score in the 'as output' model as depicted in Figure 5. Thus, it should be noted that we have to select appropriate models cautiously based on the characteristics of carry-over activity. In this case of the capital asset, it is usually treated as a "capital input" as in the 'as input' model. However, the 'as output' model will be preferred for evaluation of capital expansion policy, and the free model will be suitable for estimation of the optimal capital level.

Figures 7 and 8 exhibit respectively the averages of efficiencies of 41 US and 9 Japan companies obtained from the efficiency scores of the whole 50 companies.

<<Figure 7: Averages of 41 US DMUs out of 50 DMUs>>

<<Figure 8: Averages of 9 Japanese DMUs out of 50 DMUs >>

Comparing Figure 7 with Figure 8, we can see that the growing trend in Figure 2 of 50 companies can be largely attributed to the improvement of US companies, whereas Japanese

\footnotetext{
${ }^{1}$ Usually, the productivity index is calculated as output divided by input. However, in Figures 4 and 6, they are inverted, because in our case it is suitable to show the overuse or shortfall of capital inputs relative to the output.
} 
companies are almost saturated in efficiency as trend.

\subsection{Comparison with the separate model}

As we mentioned earlier, the dynamic model provides the efficiency index that takes into account a long view during the study period. In Figure 9, the overall efficiency of dynamic models for the DMU C are compared with that of the traditional model, which is so-called a "separate model" for measuring efficiency scores year by year independently. ${ }^{2}$ The results of the 'as input' cases are very similar between the dynamic and separate models.

\section{<<Figure 9: Comparison of overall efficiencies of DMU C between the dynamic and separate models>>}

However, focusing on factor efficiency for the capital input, we can find large differences (Figure 10). Here, the factor efficiency index (FEI) is measured for each factor as

$$
F E I=\frac{\text { Actual Data }}{\text { Projection }}-1
$$

FEI indicates the relative difference from the optimal value (projection). If it is closer to zero, the DMU is more efficient, while positive and negative values imply excess and shortfall against the optimal value, respectively.

In addition, Figure 11 depicts trends of capital input, output, and CPI (output/capital input), which are standardized by the average, respectively.

\section{$<<$ Figure 10: Comparison of factor efficiency indices for capital input of DMU C between the dynamic and separate models>>}

\section{<<Figure 11: Trend of capital input, output and capital productivity index for DMU C>>}

The capital input of DMU C is nearly flat during the study period, in spite of increasing the electric power production (output), so that CPI is improving (Figure 11). Corresponding to the upward trend of CPI, FEI scores of the separate model is getting closer to zero, i.e. becoming efficient (Figure 10). Focusing on the first two years, they exhibit considerably higher scores than those of the other periods, which imply over-investment of capital input.

The separate model is calculated year by year independently, and thus, the result is much influenced by CPI in each year. However, company managers invest in their power plants looking

\footnotetext{
${ }^{2}$ In a considerable number of previous studies, the capital variable was treated as input (see [9]). Therefore, in this figure, we depicted results of 'as input' and "free." 'As output' models provide similar results, although we did not show here.
} 
ahead the future growth of electricity demands and it would be irrelevant to evaluate the over-investment as inefficient on a single-year basis. Compared to the separate model, the dynamic model takes into account CPIs of the whole years during the study period. As the result, FEIs of the dynamic models in 1990 and 91 are much closer to the optimal values than those of the separate model, in which they are evaluated as over-investment. This would demonstrate the adaptability of the dynamic model.

Figures 12 and 13 are for the DMU D. While the capital input is flat except 1996, electric power production is volatile in Figure 13, which could be caused by the fluctuation of electric demands. In Figure 12, FEI of the separate model also exhibits volatility in line with CPI in Figure 13. However, it is impractical to change the amount of the capital assets quickly. Compared with this, the results of dynamic models demonstrate smoother behaviors from 1990 to 95, and are more reasonable and acceptable.

<<Figure 12: Comparison of factor efficiency indices for capital input of DMU D between the dynamic and separate models>>

$<<$ Figure 13: Trend of capital input, output and capital productivity index for DMU D>>

\section{Concluding remarks}

In this paper, we have proposed a slacks-based dynamic DEA (DSBM) model and applied it to electric utilities. As an SBM model, DSBM is non-radial and can deal with inefficiency of inputs and/or outputs individually as contrast to the radial models which assume proportional changes of inputs or outputs. Furthermore, we categorized carry-over activities into four types, good, bad, free and fixed.

Then, we applied these models to the electric utilities in the U.S. and Japan. In this study, the capital input is treated as a carry-over link variable, because utilities invest in their facilities to cover the future growth of electric demands and the capital investment is not for the sake of single year.

The results indicated that the classification of carry-over types exerted a large effect on the efficiency measurement, and thus, we should select appropriate models cautiously based on the characteristics of carry-over activity. Also we compared the results of dynamic models with those of the traditional separate model. Consequently, we proved that the dynamic models could avoid the deficits of single year evaluation models and were more practical and reasonable for taking into account the long-term investment than the separate model.

Future research subjects include:

(1) Decomposition of inefficiency into input, output and link inefficiencies 
(2) Dynamic cost, revenue and profit efficiencies

(3) Dual structure and shadow prices of links

(4) Evaluation of frontier-shift effects in Dynamic SBM

(5) Further comparisons with other methods, e.g. Window analysis and Malmquist index and

(6) Extensions to "Dynamic and Network" SBM models

\section{References}

[1]P. Bogetoft, R. Färe, S. Grosskopf, K. Hayes and L. Taylor, Network DEA: some applications and illustrations, Proceedings of DEA Symposium 2008 (Feb. 2008).

[2]A. Charnes, T. Clark, W.W. Cooper and B. Golany, A developmental study of data envelopment analysis in measuring the efficiency of maintenance units in the U.S. Air Forces, Annals of Operations Research 2(1) (1985).

[3] EIA, Form EIA-860: Annual Electric Generator Report, Energy Information Administration, 1990-1996 (each year).

[4]R. Färe and S. Grosskopf, Intertemporal Production Frontiers (Kluer, Norwell, 1996).

[5]R. Färe, S. Grosskopf, S. Norris and Z. Zhang, Productivity growth, technical progress, and efficiency change in industrialized countries, The American Economic Review 84(1) (1994).

[6] FEPC, Handbook of Electric Power Industry, Federation of Electric Power Companies, 1990-1996 (each year). (in Japanese).

[7] FERC, FORM No.1: Annual Report of Major Electric Utility, Federal Energy Regulatory Commission, 1990-1996 (each year).

[8] FERC, FORM No.423: Cost and Quality of Fuels for Electric Plants, Federal Energy Regulatory Commission, 1990-1996 (each year).

[9] T. Jamasb and M. Pollitt, Benchmarking and regulation: international electricity experience, Utilities Policy 9(3) (2000).

[10] C. Kao, Network data envelopment analysis: current development and future research, Asia-Pacific Productivity Conference (APPC) 2008 (Jul. 2008).

[11]G.A. Klopp, The analysis of the efficiency of production system with multiple inputs and outputs, Ph.D. dissertation, University of Illinois at Chicago, Industrial and System Engineering College (1985).

[12] S. Malmquist, Index numbers and indifference surfaces, Trabajos de Estadistica 4(1) (1053).

[13] J. Nemoto and M. Goto, Dynamic data envelopment analysis modeling intertemporal behavior of a firm in the presence of productive inefficiencies, Economic Letters, 64(1) (1999).

[14] J. Nemoto and M. Goto, Measuring dynamic efficiency in production: An application of data envelopment analysis to Japanese electric utilities, Journal of Productivity Analysis, 19(2-3) (2003). 
[15] J.T. Pastor, J.L. Ruiz and I. Sirvent, An enhanced DEA Russell graph efficiency measure, European Journal of Operational Research 115(3) (1999).

[16] T. Sueyoshi and K. Sekitani, Returns to scale in dynamic DEA, European Journal of Operational Research 161(2) (2005).

[17] K. Tone, A slacks-based measure of efficiency in data envelopment analysis, European Journal of Operational Research 130(3) (2005).

[18] K. Tone and M. Tsutsui, Network DEA: A slacks-based measure approach, European Journal of Operational Research. doi: 10.1016/j.ejor. 2008.05.027. (2008). 


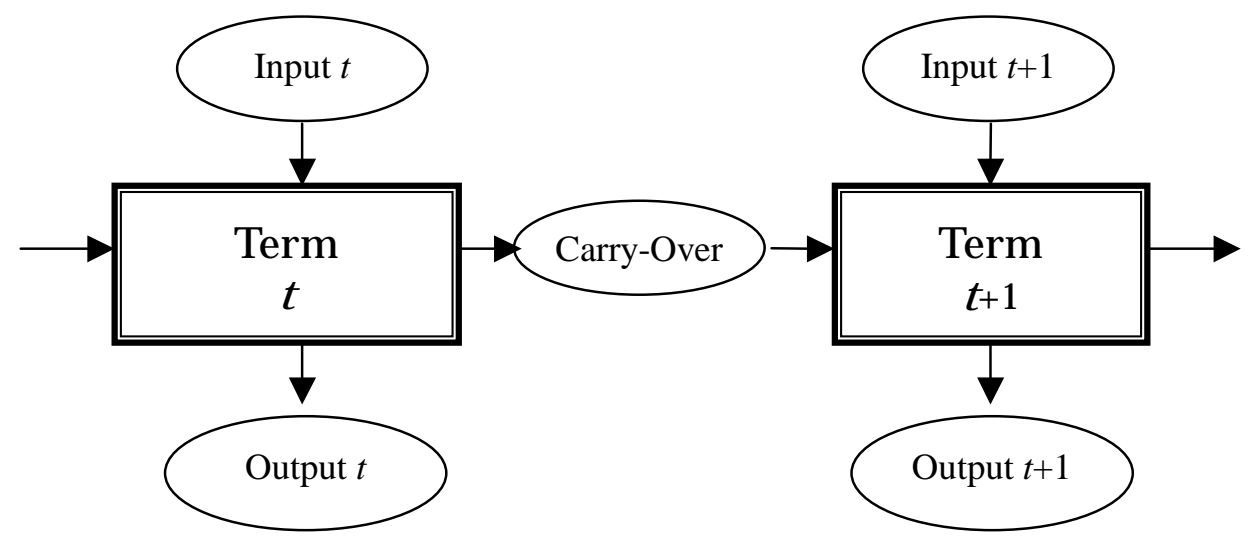

Figure 1: Dynamic structure 
Table 1: Statistics of data set

\begin{tabular}{|c|c|c|c|c|c|c|c|c|}
\hline & \multicolumn{4}{|c|}{ US } & \multicolumn{4}{|c|}{$\mathbf{J P}$} \\
\hline & Input1 & Input2 & Output & Link & Input1 & Input2 & Output & Link \\
\hline & $\begin{array}{c}\text { Employe } \\
\text { e } \\
(\#)\end{array}$ & $\begin{array}{c}\text { Fuel } \\
\left(10^{9} \mathrm{BTU}\right)\end{array}$ & $\begin{array}{c}\begin{array}{c}\text { Generated } \\
\text { Power } \\
\text { (GWh) }\end{array} \\
\end{array}$ & $\begin{array}{c}\text { Capital } \\
\text { Asset } \\
\text { (MW) }\end{array}$ & $\begin{array}{c}\text { Employe } \\
\text { e } \\
(\#) \\
\end{array}$ & $\begin{array}{c}\text { Fuel } \\
\left(10^{9} \mathrm{BTU}\right)\end{array}$ & $\begin{array}{c}\text { Generated } \\
\text { Power } \\
\text { (GWh) }\end{array}$ & $\begin{array}{c}\text { Capital } \\
\text { Asset } \\
\text { (MW) }\end{array}$ \\
\hline Average & 3,044 & 328,290 & 32,282 & 8,557 & 3,082 & 656,234 & 72,036 & 18,031 \\
\hline S.D. & 2,433 & 213,425 & 19,884 & 5,306 & 2,038 & 622,821 & 63,178 & 14,860 \\
\hline MAX & 12,155 & $1,055,926$ & 94,997 & 24,433 & 7,375 & $2,309,568$ & 232,516 & 53,975 \\
\hline MIN & 223 & 43,377 & 5,044 & 1,487 & 1,062 & 73,373 & 14,292 & 3,956 \\
\hline
\end{tabular}




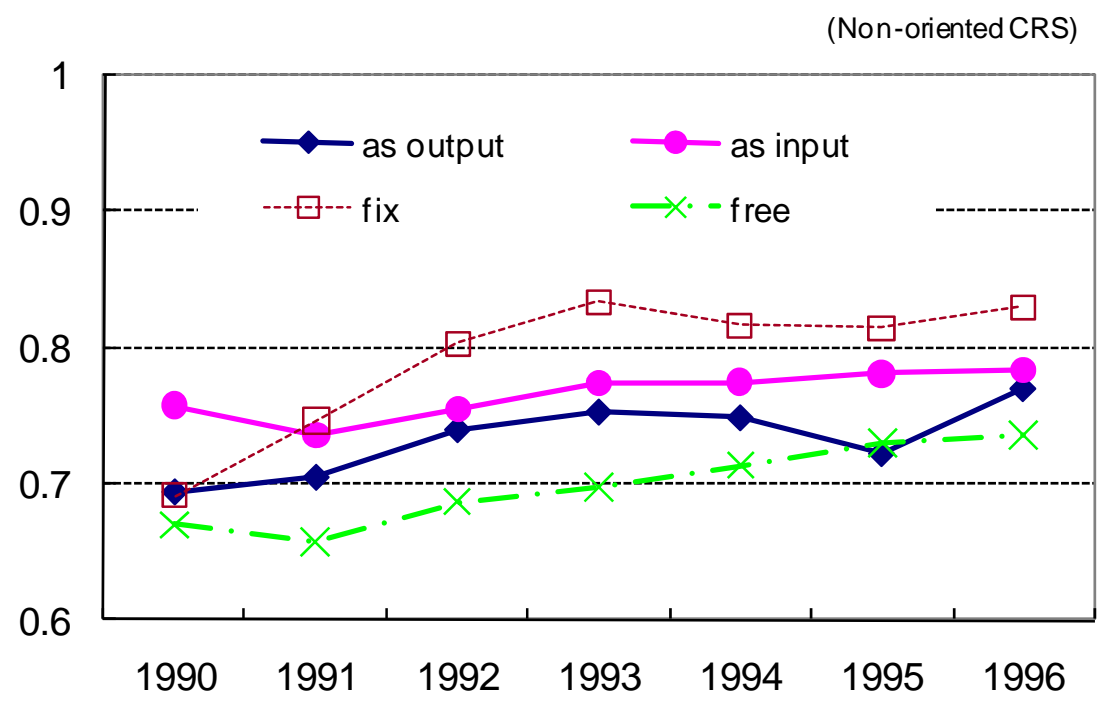

Figure 2: Averages of efficiency change of 50 US and Japanese DMUs 


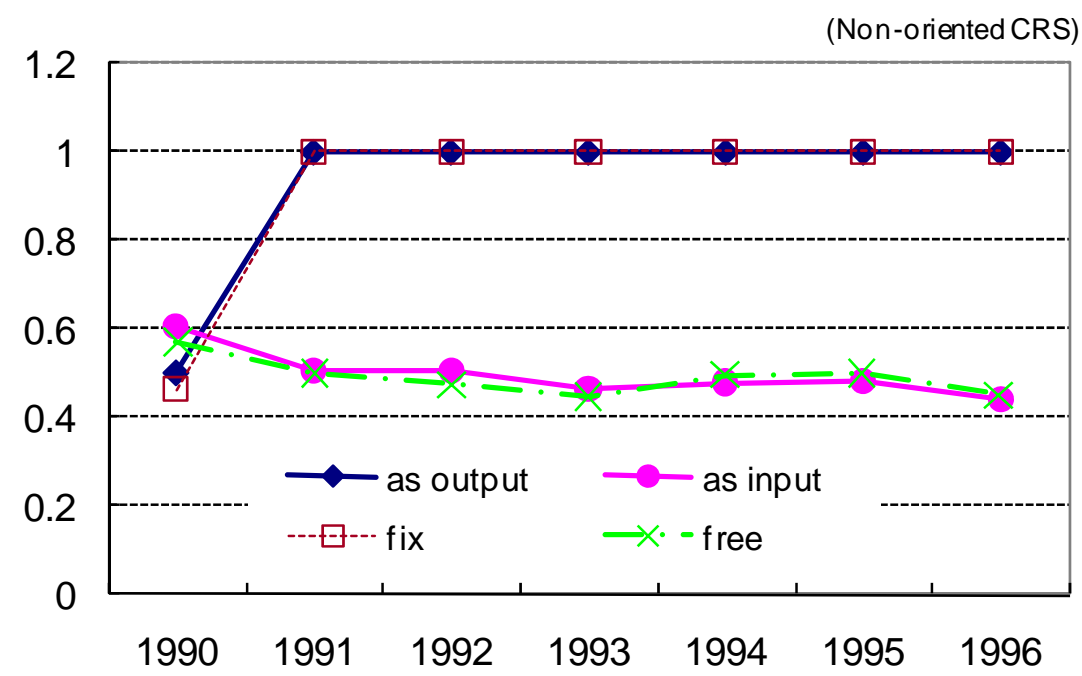

Figure 3: Efficiency change of DMU A 


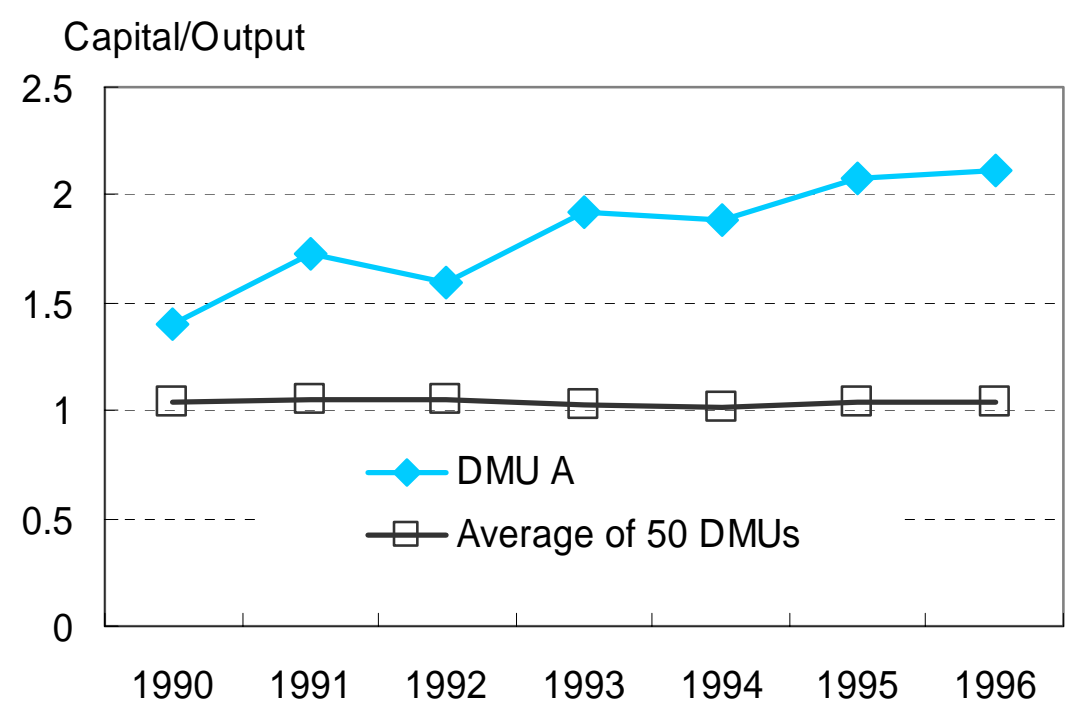

Figure 4: Comparison of the inverse of capital productivity index between DMU $A$ and the average of 50 DMUs 


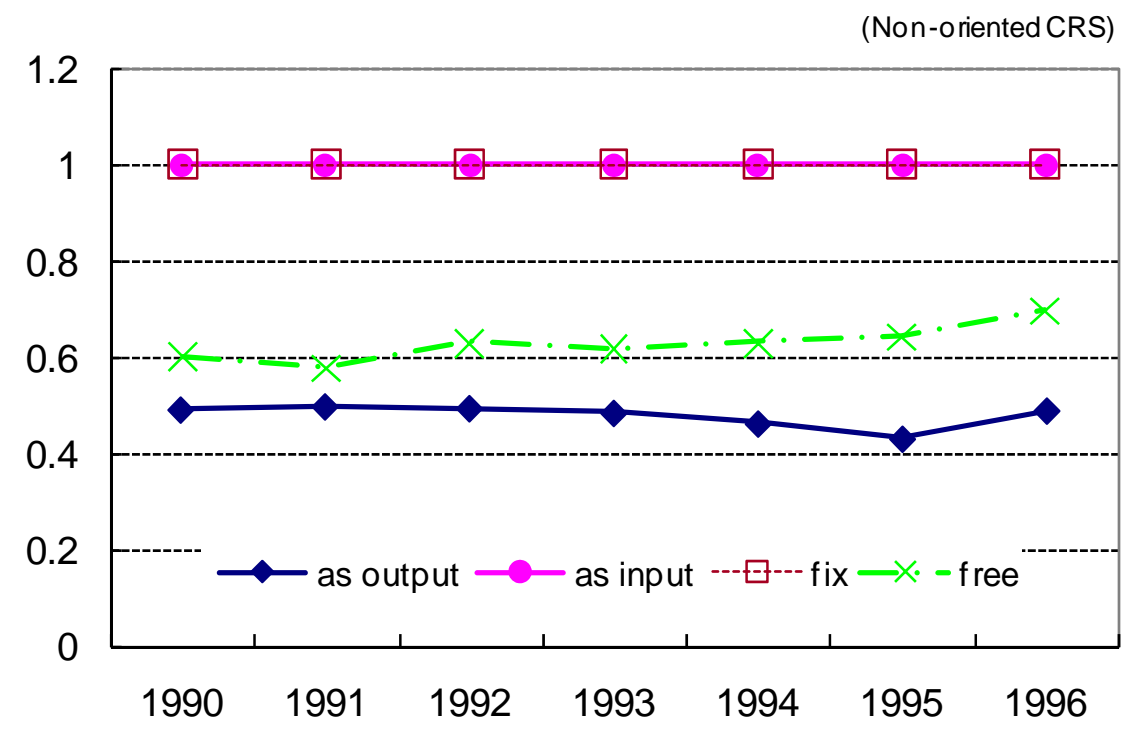

Figure 5: Efficiency change of DMU B 


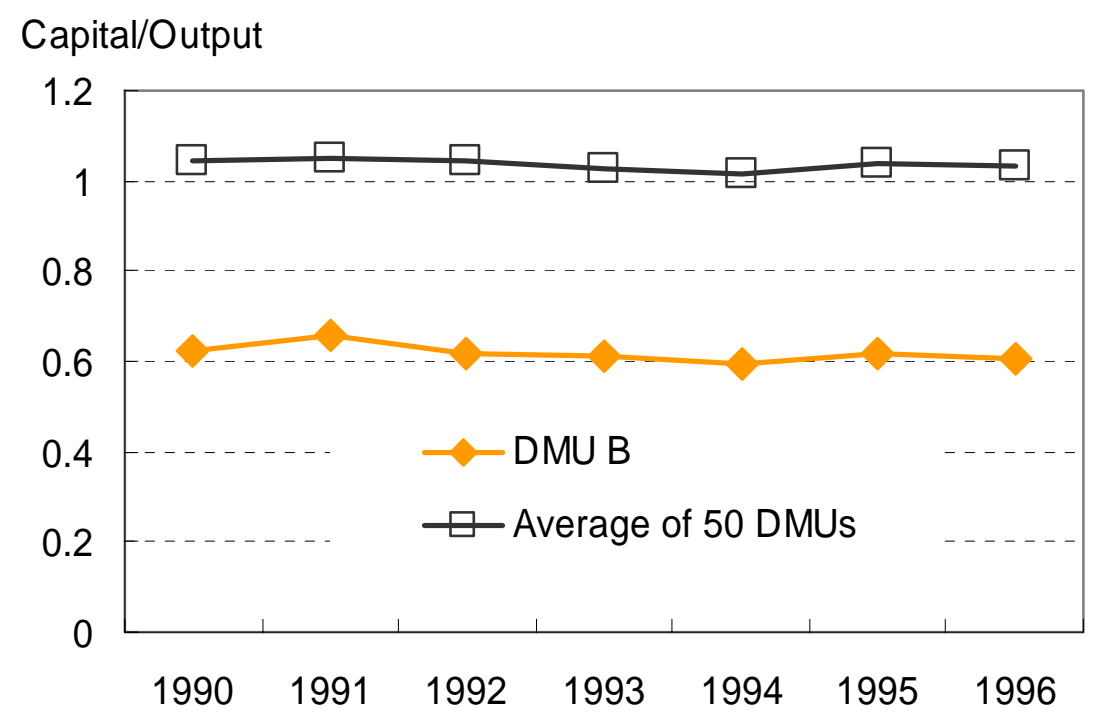

Figure 6: Comparison of the inverse of capital productivity index between DMU $B$ and the average of 50 DMUs 
(Non-oriented CRS)

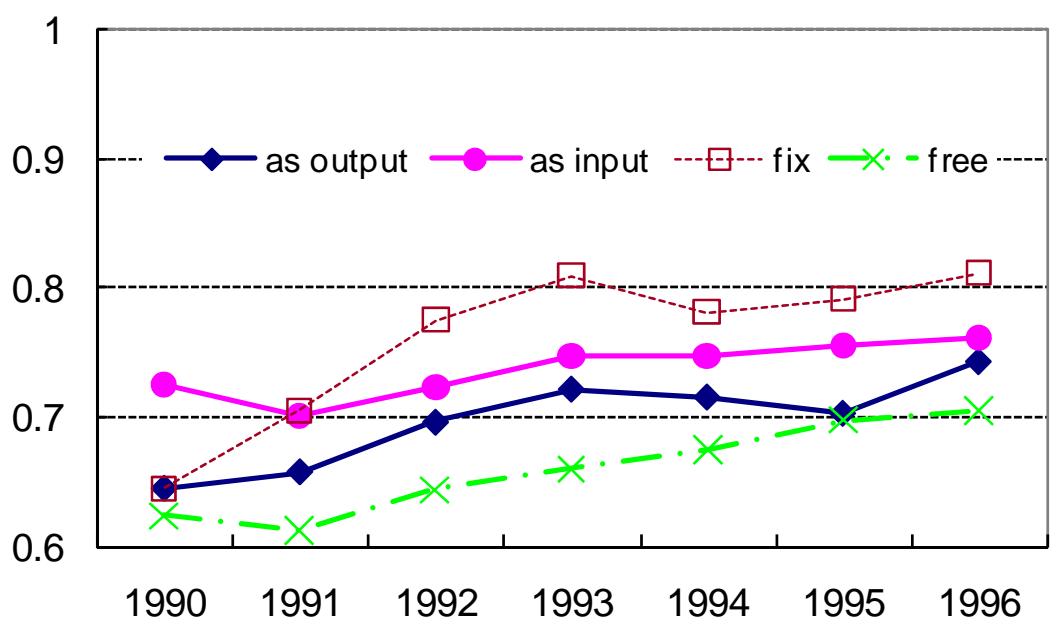

Figure 7: Averages of 41 US DMUs out of 50 DMUs 


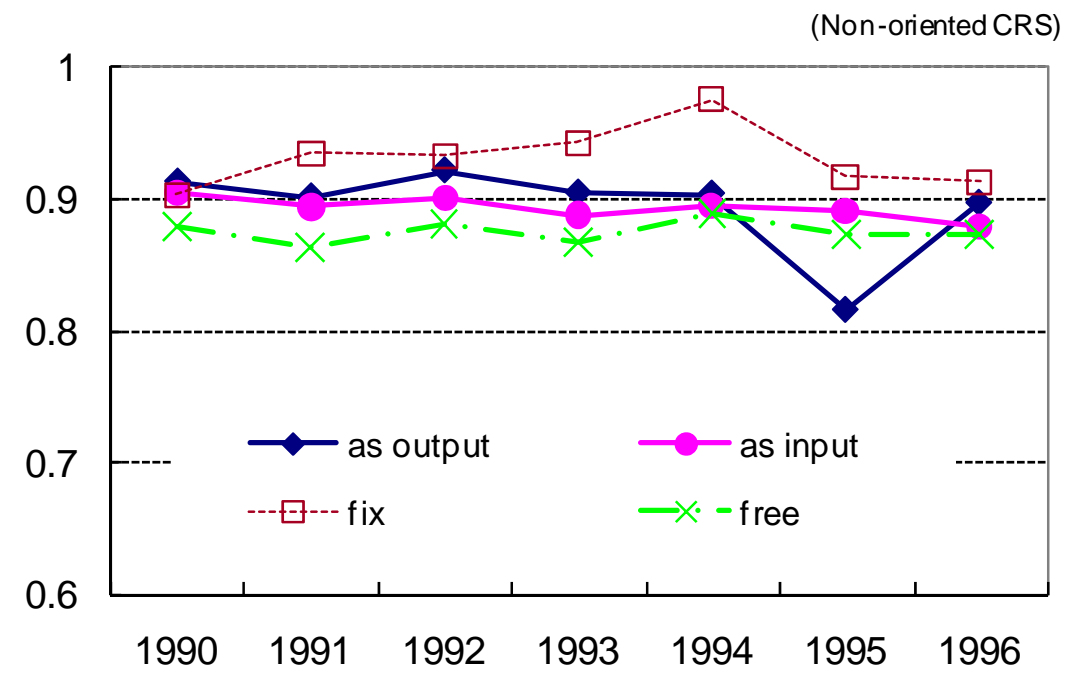

Figure 8: Averages of 9 Japanese DMUs out of 50 DMUs 
(Non-oriented CRS)

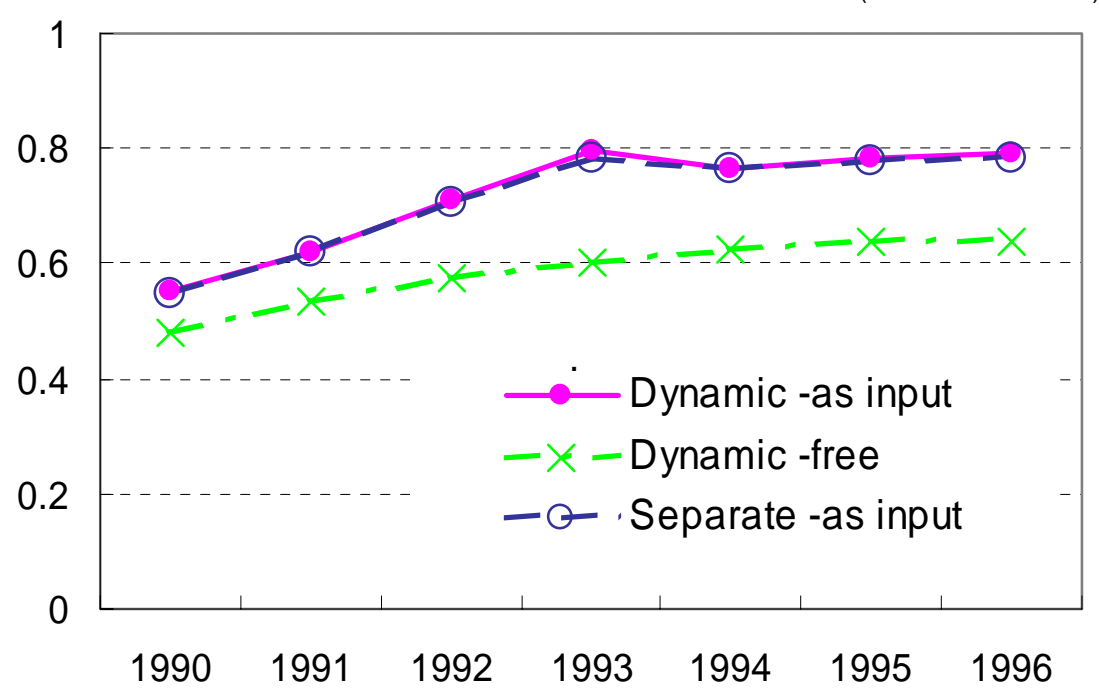

Figure 9: Comparison of overall efficiencies of DMU C between the dynamic and separate models 
(Non-oriented CRS)

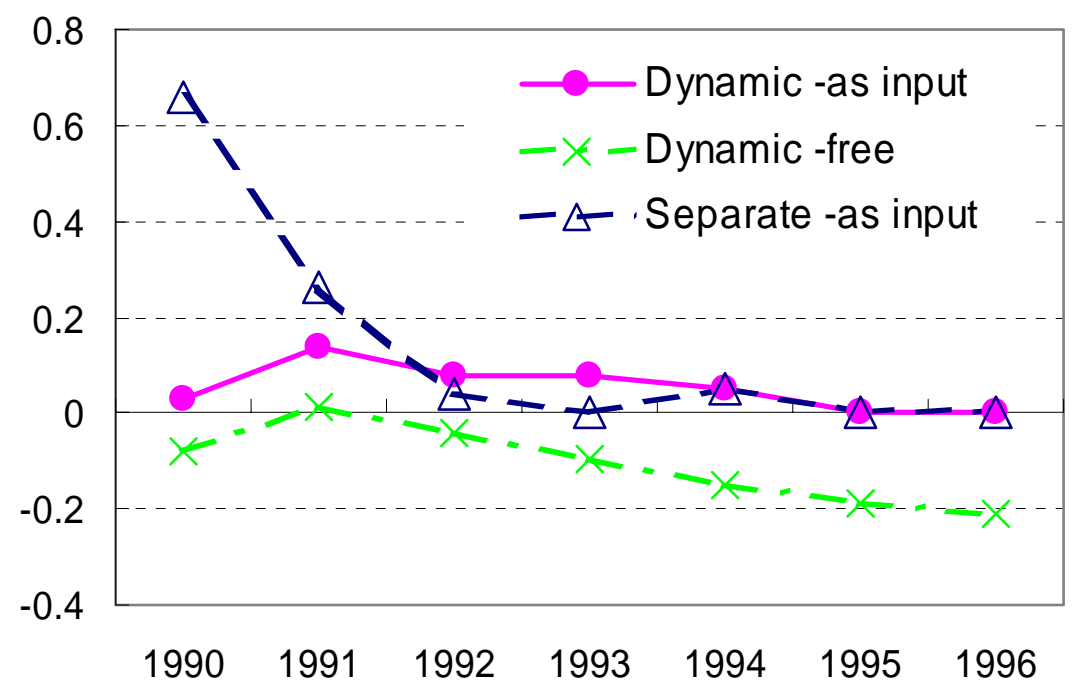

Figure 10: Comparison of factor efficiency indices for capital input of DMU C between the dynamic and separate models 


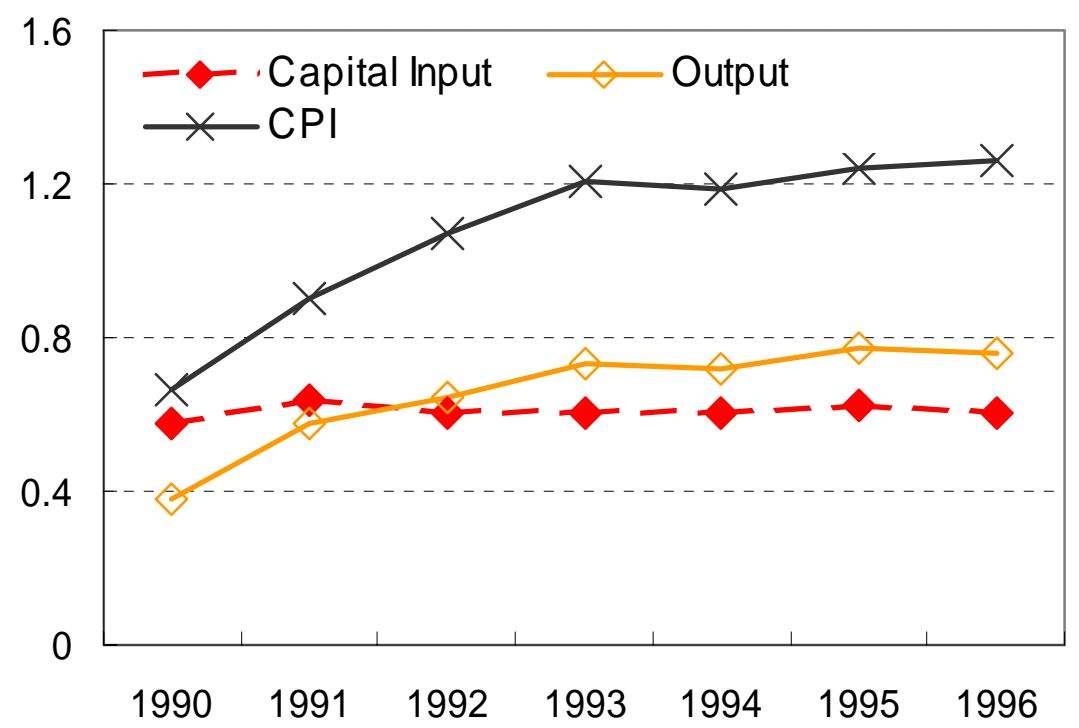

Figure 11: Trend of capital input, output and capital productivity index for DMU C 


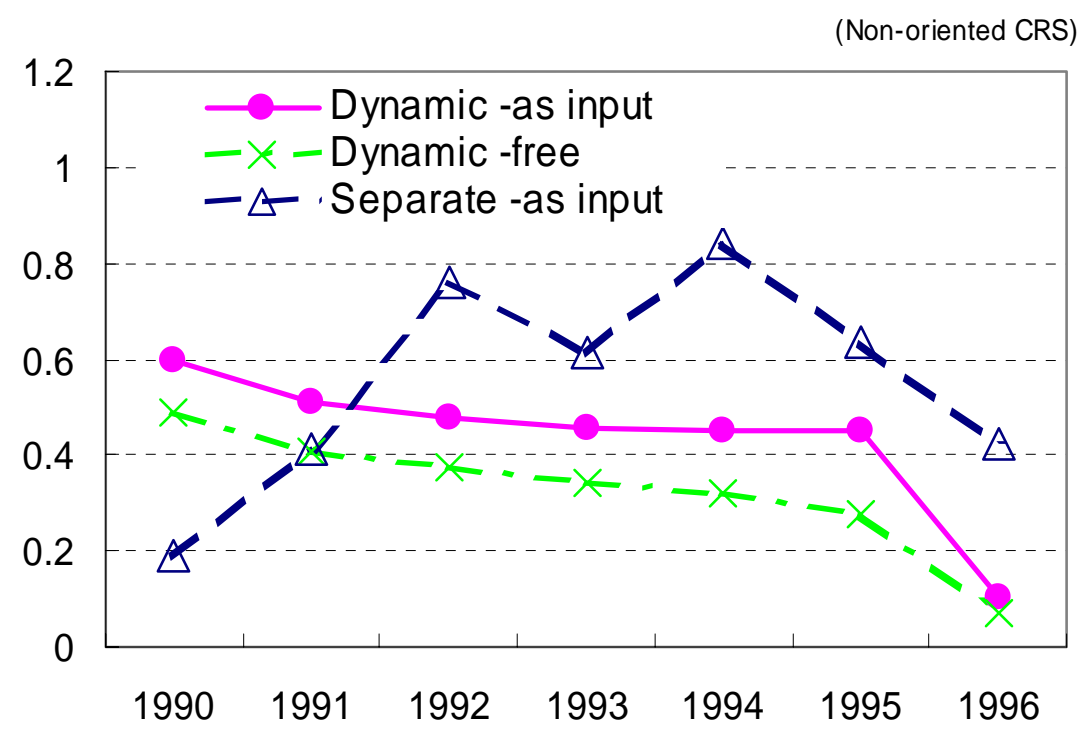

Figure 12: Comparison of factor efficiency indices for capital input of DMU D between the dynamic and separate models 


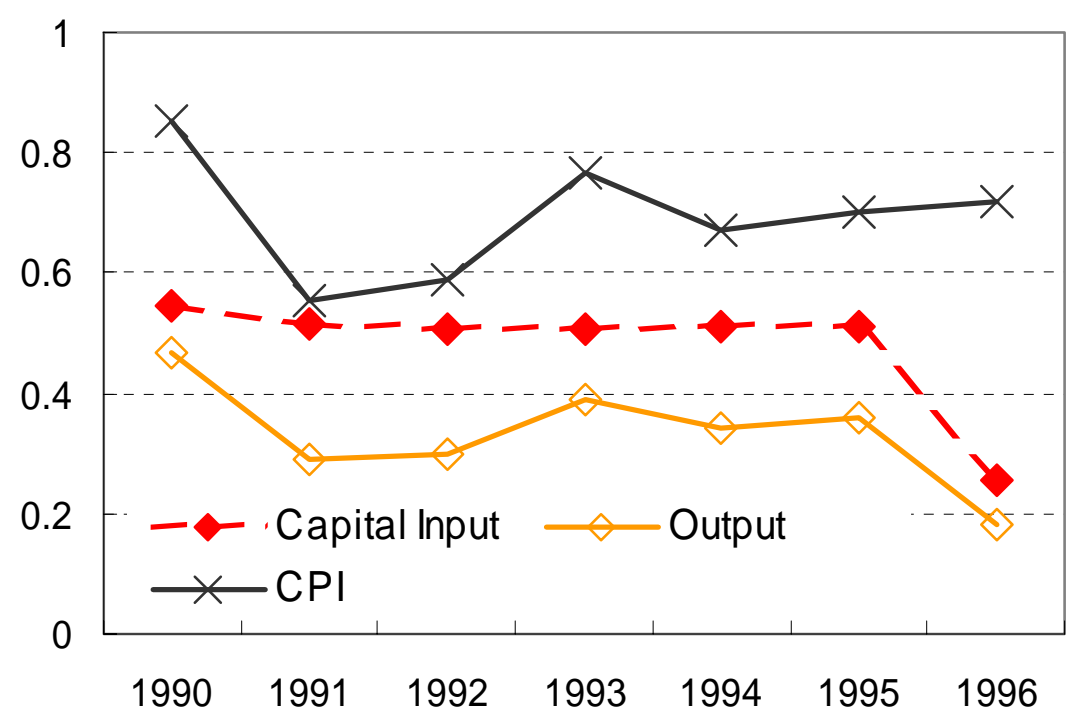

Figure 13: Trend of capital input, output and capital productivity index for DMU D 